\title{
Place des lipides dans I'alimentation du nourrisson
}

\author{
Philippe GUESNET ${ }^{1}$ \\ Gérard AILHAUD ${ }^{2}$ \\ Bernadette DELPLANQUE ${ }^{3}$ \\ Jean-Marc ALESSANDRI ${ }^{4}$ \\ ${ }^{1}$ PG Consulting, \\ 13 villa Bellevue, \\ Bures sur Yvette, \\ France \\ $<$ guesnet07@sfr.fr> \\ 2 Université de Nice Sophia-Antipolis, \\ Nice, \\ France \\ ${ }^{3}$ UMR 8195 CNRS - Centre de \\ Neurosciences Paris-Sud, \\ NMPA - Neuroendocrinologie \\ Moléculaire de la Prise Alimentaire, \\ Université Paris-Sud XI, \\ Orsay, \\ France \\ ${ }^{4}$ NuRéliCe, \\ INRA, \\ Jouy-en-Josas, \\ France
}

Article reçu le 17 janvier 2013

Accepté le 25 janvier 2013

La croissance rapide du nourrisson au cours de la première année de vie nécessite des apports énergétiques très élevés. Rapportés au poids corporel, ces apports sont 4 à 5 fois supérieurs à ceux d'un homme adulte (environ $100 \mathrm{kcal} / \mathrm{kg} /$ J). Ils sont couverts par la densité calorique particulièrement élevée du lait humain (650-700 kcal/L). Ce dernier renferme en effet des concentrations exceptionnellement élevées de glucides et surtout de lipides $(37 \mathrm{~g} / \mathrm{L})$, présents très majoritairement sous la forme de triglycérides (>98\%). Ainsi, un nourrisson à l'âge de 1 mois ingère près de $30 \mathrm{~g}$ de triglycérides par jour, ce qui correspond à une alimentation hyperlipidique car,

\begin{abstract}
Lipids in infant nutrition
The rapid growth of the neonate requires a high intake of both energy and lipids. Fatty acids may play an important role in the brain development and in the etiology of several disorders in children and adults. Most of infant formulas currently marketed have a fatty acid profile globally mimicking that of the "gold reference", the human milk. However several issues remain to be improved to optimizing their lipid status:

Structure of the fat globule. Fat globules of human milk differ from those of infant formulas by their larger size and the presence of a phospholipid membrane. Recent experimental data in animal models of infant nutrition suggest that this specificity of natural milk may prevent the development of the metabolic syndrome. In addition, palmitic acid is mainly esterified in the sn-2 position of triglycerides in human milk, versus in the sn-1 and sn-3 positions in infant formulas. This sn-2 esterification may favor the intestinal absorption of other fatty acids and then their tissue bioavailability ; PUFA metabolism. The exclusive use of vegetable oils in infant formulas has increased the content of both polyunsaturated fatty acid precursors (linoleic and $\alpha$-linolenic acids). However, several studies have led to the observation that high PUFA contents in formulas may reduce the incorporation of docosahexaenoic acid in tissues;

$\mathrm{N}-6$ PUFA intake and obesity. In western countries, the increasing incidence of obesity has coincided with the gradual increase of $n-6$ PUFA from food and, incidentally, from an $n-6 / n-3$ imbalance. Animal studies have recently shown that high linoleic acid intake and high ratio of linoleic to $\alpha$-linolenic acids during the perinatal period activate the adipose tissue growth in the young and adult. To date, two observational studies confirm the existence of a link between the $n-6 / n-3$ ratio in cord or the $n-6$ content in maternal plasma and adiposity in 3-7 years old infants.
\end{abstract}

Key words: docosahexaenoic acid, lipid droplets, linoleic acid, $\alpha$-linolenic acid, newborn infant, obesity

rapporté au poids corporel, ce niveau de consommation est 3 à 5 fois plus élevé que celui d'un homme adulte (Innis, 2011).

L'allaitement maternel est recommandé au cours des six premiers mois de vie (Salle, 2009), car en plus de la couverture des besoins nutritionnels du nouveau-né, il exerce un éventail d'effets bénéfiques sur le développement neurosensoriel des enfants et leur santé (prévention de dermatose atopique, d'infection, de surpoids, etc. (Beyerlein et von Kries, 2011 ; Kramer, 2011). L'allaitement maternel jouerait également un rôle important pour la santé à l'âge adulte en réduisant l'incidence de pathologies métaboliques liées à l'obésité, les maladies cardiovasculaires, I'hypertension et I'hypercholestérolémie (concept de programmation précoce) (Beyerlein et von Kries, 2011 ; Owen et al., 2011). La composition lipidique du lait maternel a ainsi servi de support pour déterminer les besoins physiologiques pendant les 6 premiers mois de vie, puis pour formuler les aliments lactés pour nourrissons. La teneur en AGPI du lait maternel est cependant très dépendante de l'alimentation de la mère : elle se répartit sur une large échelle de valeurs en ce qui concerne le 18:2n-6 et le rapport 18:2n6/18:3n-3 (facteur compris entre 3 et 4 ) (Guesnet et al., 1995). 
La plupart des laits infantiles actuellement commercialisés ont un profil en acides gras saturés (AGS), monoinsaturés (AGMI) et polyinsaturés (AGPI) $n-6$ et $n-3$ qui reproduit plus ou moins fidèlement les variations naturelles du lait humain. Le tableau 1 rapporte les teneurs moyennes en acides gras du lait mature de femme pour la France (Boué-Vaysse et al., 2009 ; Guesnet et al., 1993). Les AGS y constituent près de la moitié des acides gras totaux. Ils sont majoritairement représentés par les acides palmitique (16:0, $50 \%$ des AGS) et myristique (14:0, $20 \%$ ). Les lipides du lait maternel se distinguent également par la présence d'une quantité substantielle d'acides gras à chaîne moyenne $(<14: 0$, $20 \%$ ). Les AGMI représentent pour leur part environ $40 \%$ des acides gras totaux du lait, l'acide oléique étant le représentant majoritaire de cette famille (18:1n-9, $75 \%$ des AGMI). Parmi les AGPI n-6, I'acide linoléique (18:2n-6) représente 11 à $13 \%$ des acides gras totaux et $90 \%$ environ des AGPI de cette série. Les AGPI n-6 à longue chaîne sont représentés principalement par l'acide arachidonique (20:4 n-6), dont la teneur est de l'ordre de 0,4\%. Dans la famille n3 , I'acide $\alpha$-linolénique (18:3n-3) occupe une place aussi prépondérante que celle du 18:2n-6 dans la famille n-6. Avec une teneur de 0,7-1 \% des acides gras totaux, cet AGPI représente $70 \%$ des AGPI n-3 totaux. Deux tiers des AGPI à longue chaîne $n-3$ sont sous forme d'acide docosahexaénoïque (22:6n-3, DHA) qui représente $0,2-0,3 \%$ des acides gras totaux. Enfin, le rapport 18:2n-6/18:3n-3 est généralement compris entre 12 et 15 . En termes de santé du nourrisson et du jeune enfant, plusieurs aspects restent encore à explorer pour définir un profil lipidique optimum. Ces investigations portent sur la structure du globule gras qui est très spécifique dans le lait maternel (taille du globule et distribution du 16:0 sur le glycérol), sur la réévaluation des apports en AGPI précurseurs, sur les bénéfices neurofonctionnels à long terme d'un apport en DHA préformé, et sur l'importance de l'équilibre n-6/n-3 de la nutrition maternelle pendant la période périnatale vis-à-vis du risque d'obésité chez l'enfant.

\section{Caractéristiques structurales des globules gras du lait - Taille et position stéréospécifique de l'acide palmitique (16:0) sur le triglycéride}

Les globules gras du lait humain se distinguent de ceux des formules lactées par leur taille plus élevée et par la présence d'une membrane phospholipidique. Le diamètre modal moyen des globules gras du lait mature de femme est de l'ordre de $4 \mu \mathrm{m}$ (distribution : 1-10 $\mu \mathrm{m}$ ) tandis que celui des formules est 4 fois plus petit, soit

Tableau 1. Variations des teneurs moyennes des principaux acides gras du lait maternel en France (Boué-Vaysse et al., 2009) ${ }^{7}$.

\begin{tabular}{|ll|}
\hline Acides gras & \% des acides gras totaux \\
\hline Acides gras saturés & $45,0-49,0$ \\
\hline $8: 0+10: 0+12: 0^{2}$ & $7,0-10,0$ \\
\hline $14: 0^{2}$ & $6,5-9,0$ \\
\hline $16: 0^{2}$ & $22,5-24,5$ \\
\hline Acides gras mono-insaturés & $37,0-40,0$ \\
\hline $18: 1 n-9^{2}$ & $27,5-30,5$ \\
\hline Acides gras polyinsaturés $n-6$ & $12,5-15,0$ \\
\hline $18: 2 n-6$ & $10,5-12,7$ \\
\hline $20: 4 n-6$ & $0,36-0,45$ \\
\hline Acides gras polyinsaturés $n-3$ & $1,11-1,36$ \\
\hline $18: 3 n-3$ & $0,72-0,95$ \\
\hline $22: 6 n-3$ & $0,19-0,29$ \\
\hline $18: 2 n-6 / 18: 3 n-3$ & $11,8-14,6$ \\
\hline
\end{tabular}

${ }^{1}$ Les analyses ont été réalisées sur 145 échantillons de lait maternel mature ( $>30^{\mathrm{e}}$ jour de lactation) collectées en 2007 dans plusieurs régions françaises.

${ }^{2}$ Adapté de Guesnet et al. (1993). en moyenne 0,4 $\mu \mathrm{m}$ (distribution : 0,3$1 \mu \mathrm{m}$ ) (Armand et al., 1996 ; Michalski et al., 2005a). Par ailleurs, les globules gras du lait maternel sont entourés d'une membrane provenant des cellules épithéliales mammaires et dont les constituants sont ceux qui composent les membranes biologiques (phospholipides, cholestérol libre, glycoprotéines, protéines et enzymes). Au contraire, les globules des formules sont entourés de protéines du lait (de vache) qui adhèrent à leur surface (Michalski et al., 2005a). Des données expérimentales récentes suggèrent que la taille et la structure des globules gras du lait seraient des paramètres déterminants dans les effets bénéfiques à long terme de l'allaitement maternel, notamment en ce qui concerne la protection contre le syndrome métabolique et l'obésité (Oosting et al., 2012). Pendant la période de développement chez la souris (du sevrage au stade jeune adulte), la consommation d'un régime lacté composé de globules lipidiques de grande taille $(10 \mu \mathrm{m})$ et entourée d'une membrane phospholipidique réduit de $25 \%$ la masse totale de tissu adipeux et la teneur circulante en leptine à l'âge adulte, comparativement à un régime dont la structure des gouttelettes est caractéristique de celle des laits infantiles (Oosting et al., 2012). Cet effet anti-obésité s'accompagne également d'une amélioration du statut métabolique de I'animal et plus spécifiquement de la sensibilité à l'insuline (réduction de I'index HOMA, de la glycémie à jeun et du taux circulant de résistine). Les auteurs émettent I'hypothèse qu'une modification des cinétiques d'absorption et de digestion des lipides peut altérer en retour leur utilisation métabolique (équilibre entre le catabolisme par $\beta$-oxydation et la lipogenèse). L'absorption des globules gras de petite taille $(0,4 \mu \mathrm{m})$ formés avec de la caséine adsorbée provoque chez le rat une diminution marquée de la $\beta$ oxydation post-prandiale des acides gras, comparativement à des globules natifs (Michalski et al., 2005b). Celle-ci résulterait d'un retard dans la cinétique d'apparition des triglycérides dans le plasma lié à un ralentissement de la vidange et de la lipolyse gastrique (Borel et al., 1994 ; Armand et al., 1996, 1999 ; Michalski et al., 2006).

La structure des lipides du lait maternel se distinguent en outre de celle des laits infantiles par l'estérification préférentielle de l'acide palmitique en position 


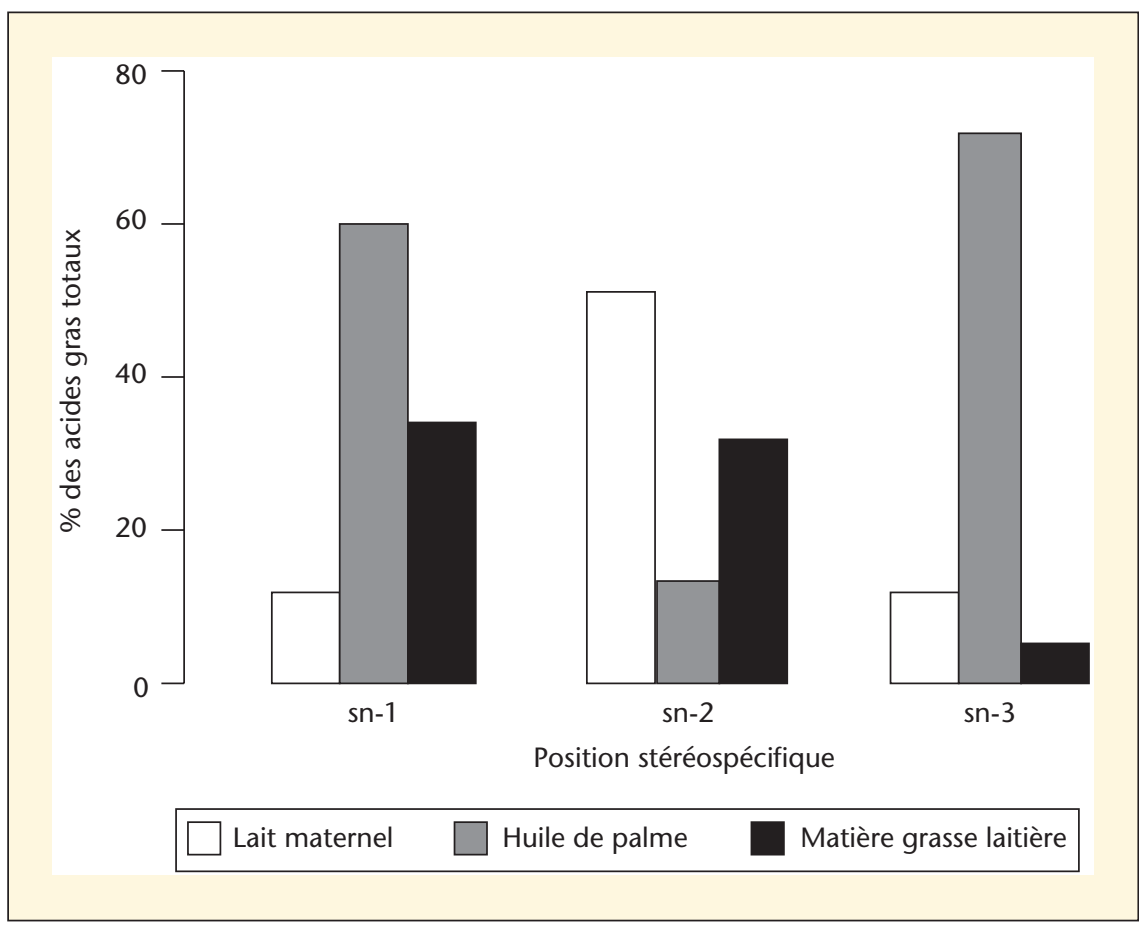

Figure 1. Distribution stéréospécifique de l'acide palmitique (16:0) sur les triglycerides du lait maternel - Comparaison avec I'huile de palme et la matiere grasse laitiere (adapté d'Innis, 2011).

sn-2 des triglycérides (Innis, 2011). En effet, près de $70 \%$ du 16:0 occupe cette position interne contre seulement $15 \%$ pour chacune des positions externes (sn-1 et sn-3) (figure 1). En revanche, dans les huiles végétales utilisées dans la formulation des laits infantiles, le 16:0 est surtout distribué sur les positions externes. Ainsi, dans I'huile de palme, on retrouve seulement $9 \%$ de cet acide gras saturé sur la position $s n-2$, contre $41 \%$ et $50 \%$ respectivement sur les positions $s n-1$ et $s n-3$ (figure 1). La distribution du 16:0 dans les matières grasses laitières est intermédiaire car $40 \%$ sont retrouvés en $s n-1$ et en $s n-2$. II est bien établi que la position stéréospécifique interne occupée par le 16:0 exerce un rôle important dans le processus d'absorption des acides gras et de minéralisation osseuse (Innis, 2011). Plusieurs études ont montré, aussi bien chez le nourrisson que chez I'animal, que la consommation d'aliments lactés apportant les acides gras saturés sous la forme d'huile ou d'oléine de palme provoquait une diminution marquée du taux d'absorption du 16:0, diminution pouvant atteindre plus de $30 \%$ chez l'enfant prématuré (Carnielli et al., 1995). Cette diminution résulte d'une excrétion fécale plus élevée de 16:0 sous la forme de savons insolubles (savons de calcium) après sa libération des positions externes des triglycérides par action de la lipase pancréatique. La perte fécale de calcium se traduit par une diminution significative de la minéralisation et de la densité osseuse chez le nourrisson (Kennedy et al., 1999). L'utilisation spécifique de triglycérides de synthèse renfermant préférentiellement du 16:0 en position interne (lipides structurés) permet de retrouver les caractéristiques nutritionnelles du lait maternel (Kennedy et al., 1999).

Récemment, il a été suggéré que la position sn-2 occupée par le 16:0 favoriserait également les processus d'absorption des acides gras au niveau intestinal ainsi que leur biodisponibilité au niveau tissulaire (Innis, 2011). Au niveau intestinal, les processus de synthèse des triglycérides et des lipoprotéines (chylomicrons) et de leur sécrétion seraient impliqués. Sur différents modèles in vitro de barrière intestinale (cellules Caco-2 et lignée cellulaire de porcelet IPEC-1) (van Greevenbroek et al., 1995 ; Yao et al., 2002), il a été montré que les acides gras saturés libres (16:0 et 18:0) inhibent la sécrétion de l'apoB et des VLDL au niveau basolatéral, en altérant notamment le processus de translocation des trigycérides du réticulum endoplasmique vers le Golgi. À l'opposé, l'acide oléique libre, qui est préférentiellement estérifié sur les positions externes des triglycérides et est donc libéré au cours de la digestion pancréatique, constituerait un facteur facilitant l'assemblage des chylomicrons et leur sécrétion. Enfin, il est à noter que la position préférentielle $s n-2$ du 16:0 dans les triglycérides du lait maternel est préservée après digestion et absorption intestinale, puisque près de $40 \%$ des acides gras totaux occupant la position interne des triglycérides circulants sont sous la forme de 16:0 (Innis et al., 1995) (figure 2). Cette préservation est reproduite avec des triglycérides structurés mimant la distribution du lait maternel. Elle pourrait ainsi modérer les effets délétères des acides gras saturés libres au niveau des tissus périphériques comme les tissus adipeux et musculaires (activité inflammatoire du 16:0) (Innis, 2011).

\section{Optimisation des apports en AGPI précurseurs $(18: 2 n-6,18: 3 n-3)$
et en acide
docosahexaénoïque
$(22: 6 n-3)$}

Chez le nouveau-né nourri uniquement au biberon, il a été observé que $1 \%$ de l'apport énergétique en acide linoléique évitait l'apparition des symptômes physiologiques (dessèchement et épaississement de la peau, desquamation) et biochimique (teneurs circulante élevée en acide eicosatriénoïque (20:3n9) et réduite en acide arachidonique) qui sont caractéristiques d'une déficience alimentaire en AGPI n-6 (Holman et al., 1970). En France, un apport minimum en $18: 2 n-6$ de $2,7 \%$ de l'énergie (6\% des acides gras totaux du lait) a été retenu (ANC acides gras, 2011). Il a été par ailleurs proposé une limite supérieure d'apport en cet AGPI (11\% de l'énergie soit environ $25 \%$ des acides gras totaux) en s'appuyant sur les teneurs les plus élevées trouvées dans le lait humain (Jensen, 1996; Ailhaud et al., 2006). L'importance nutritionnelle de l'acide $\alpha$-linolénique $(18: 3 n-3)$ repose surtout sur le fait que son principal dérivé métabolique à longue chaîne, le DHA, exerce des effets favorables sur les fonctions cérébrales et visuelles (Alessandri et al., 2004). La période de développement périnatal, qui correspond à la phase de croissance rapide 


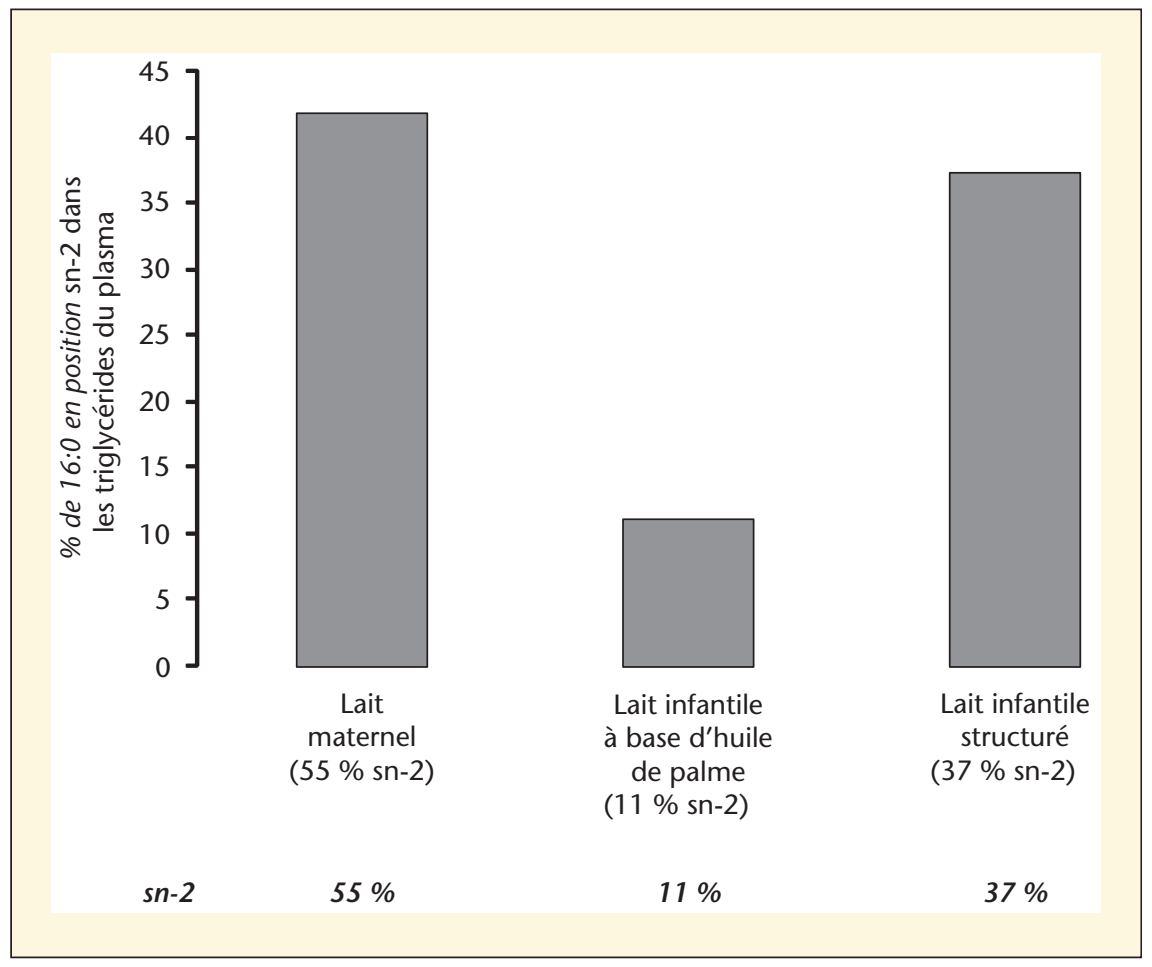

Figure 2. Pourcentage d'acide palmitique (16:0) retrouvée en position sn-2 des triglycérides plasmatiques chez le porcelet allaité ayant reçu du lait maternel, un lait classique ou un lait structuré mimant la distribution stéréospécifique du lait maternel (Innis et al., 1995).

du cerveau, est aussi la plus active pour l'incorporation du DHA. C'est donc durant cette période de la vie que la qualité des apports alimentaires en $18: 3 n-3$ - mais également en AGPI n-6 (rapport $n-6 / n-3$ ) - détermine le niveau d'incorporation du DHA (Alessandri et al., 2004). Ce sont tout d'abord les études animales, se basant sur la relation dose-dépendante d'incorporation du DHA dans les membranes cérébrales qui ont permis de déterminer un besoin optimum en 18:3n-3 du nourrisson allaité $(0,45 \%$ de l'énergie ingérée) (Bourre et al., 1989). Puis des études cliniques conduites dans ce domaine ont mis en évidence que la consommation de laits infantiles trop pauvres en 18:3n-3 et déséquilibrés par un apport excessif en 18:2n-6 (rapport $18: 2 n-6 / 18: 3 n-3$ de 40) provoquait chez le nouveau-né, qu'il soit prématuré ou né à terme, une diminution marquée de la concentration en DHA dans les lipides sanguins et cérébraux, ainsi qu'un retard de développement des fonctions visuelles, comparativement à des enfants allaités au sein pris comme référence nutritionnelle et fonctionnelle (Uauy et al., 1990; Guesnet et Alessandri, 2011). Des supplémenta- maintenant constant celui du 18:3n-3 ( $0,5 \%$ des calories ingérées) (Guesnet et al., 2011 ; Cunnane et Guesnet, 2011) : la présence d'acide $\alpha$-linolénique modère les symptômes physiologiques et biochimique d'une déficience en AGPI $n-6$, ce qui permet de situer le besoin minimum en $18: 2 n-6$ à un niveau 4 fois inférieur à celui initialement déterminé ( $0,5 \%$ de l'énergie alimentaire).

Par ailleurs, un certain nombre de données expérimentales suggèrent qu'une réduction des teneurs en AGPI précurseurs dans les laits infantiles actuels constituerait une option nutritionnelle intéressante pour optimiser la voie de biosynthèse du DHA (Gibson et al., 2011). En effet, ces teneurs sont assez élevées, notamment pour l'acide linoléique qui représente de 13 à $20 \%$ des acides gras totaux, voire jusqu'à $30 \%$. Cette valeur élevée résulte de la législation autorisant I'utilisation exclusive d'huiles végétales pour la formulation lipidique des laits infantiles (Ailhaud et al., 2006). Afin de maintenir un rapport équilibré entre les 2 familles d'AGPI (rapport 18:2n-6/18:3n-3 de 5 à 10), la teneur en 18:3n-3 a donc été également augmentée (entre 2 et $3 \%$ des acides gras totaux). Or, plusieurs études conduites suggèrent que de tels apports en AGPI essentiels précurseurs pourraient limiter la biosynthèse du DHA par des phénomènes d'inhibition compétitive au niveau de la voie de bioconversion des AGPI (Gibson et al., 2011). Ainsi sur la base des teneurs tissulaires animales en AGPI, il est mis en avant le concept que des apports alimentaires élevés en acide linoléique inhibent la voie de conversion de l'acide $\alpha$-linolénique en DHA par inhibition compétitive au niveau de la $\Delta 6$ désaturase (figure 3). Par ailleurs, un excès $d$ 'apport en acide $\alpha$-linolénique pourrait aussi limiter sa propre conversion en DHA car la voie de bioconversion fait intervenir à 2 reprises une étape de $\Delta 6$-désaturation et 2 substrats en $n-3$ qui sont des compétiteurs potentiels (18:3n3 et 24:5n-3) (figure 3). Très récemment, il a été également démontré que le 18:4n-3 pouvait également inhiber les étapes d'élongation du 20:5n-3 et du 22:5n-3 catalysée par I'Elovl2 (figure 3), et donc ainsi freiner le flux métabolique de la voie de bioconversion en condition d'apport élevé de 18:3n-3 (Gregory et al., 2011). En d'autres termes, Gibson et al. (2011) émettent I'hypo- 
thèse qu'une réduction des teneurs en 18:3n-3 de l'alimentation lactée du nourrisson, en maintenant un rapport $18: 2 n-6 / 18: 3 n-3$ inférieur à 5 , permettrait de favoriser la synthèse de DHA. Cette hypothèse est validée par plusieurs données expérimentales, dont des données cliniques obtenues chez des enfants nés à terme allaités artificiellement pendant 10 semaines. Comparativement à une alimentation lactée déséquilibrée en AGPI (18:2n-6/18:3n3 de 20), il apparaît clairement qu'un apport modéré à la fois en $18: 2 \mathrm{n}-6$ et en $18: 3 n-3$ (respectivement 3,5 et $1,1 \%$ des acides gras totaux du lait) est tout aussi efficace pour augmenter le statut sanguin en DHA du nourrisson qu'un apport élevé (13 et 3,3\%) (figure 4) (Clark et al., 1992). Des résultats très similaires ont été rapportés au niveau des membranes cérébrales sur un modèle animal mesurant la vitesse de rétablissement de la teneur cérébrale en DHA (Du et al., 2012). Sur ce modèle, il a également été mis en avant la supériorité nutritionnelle des matières grasses laitières par rapport à I'huile de palme. En effet, la teneur cérébrale en DHA est plus élevée chez les animaux ingérant une alimentation équilibrée en AGPI à base de beurre (Du et al., 2012 ; Delplanque et al., 2013). Les auteurs évoquent notamment la présence spécifique $d^{\prime}$ acides gras à chaîne courte (4:0 et 6:0) et moyenne $(8: 0,10: 0$ et $12: 0)$ qui sont facilement catabolisables préservant ainsi l'acide $\alpha$-linolénique d'un catabolisme excessif par $\beta$-oxydation.

En dépit des controverses sur le développement visuel et cognitif du nourrisson né à terme, le consensus actuel est en faveur d'un apport alimentaire lacté en DHA (au moins pendant les 6 premiers mois de vie) avec adjonction de DHA dans les formules à un niveau équivalent à celui du lait humain $(0,2-0,5 \%$ du poids des acides gras totaux) (Guesnet et Alessandri, 2011). II existe actuellement peu d'études ayant évalué les répercussions neurofonctionnelles à long terme ( $>1$ an) de l'apport en DHA préformé. Conduite sur 250 enfants âgés de 4 ans nés à terme et ayant reçu pendant 6 mois des laits classiques ou supplémentés en DHA (0,2-0,4\% des acides gras totaux), l'étude prospective du Southampton Women's Survey démontre bien de meilleures capacités cognitives chez les enfants allaités au

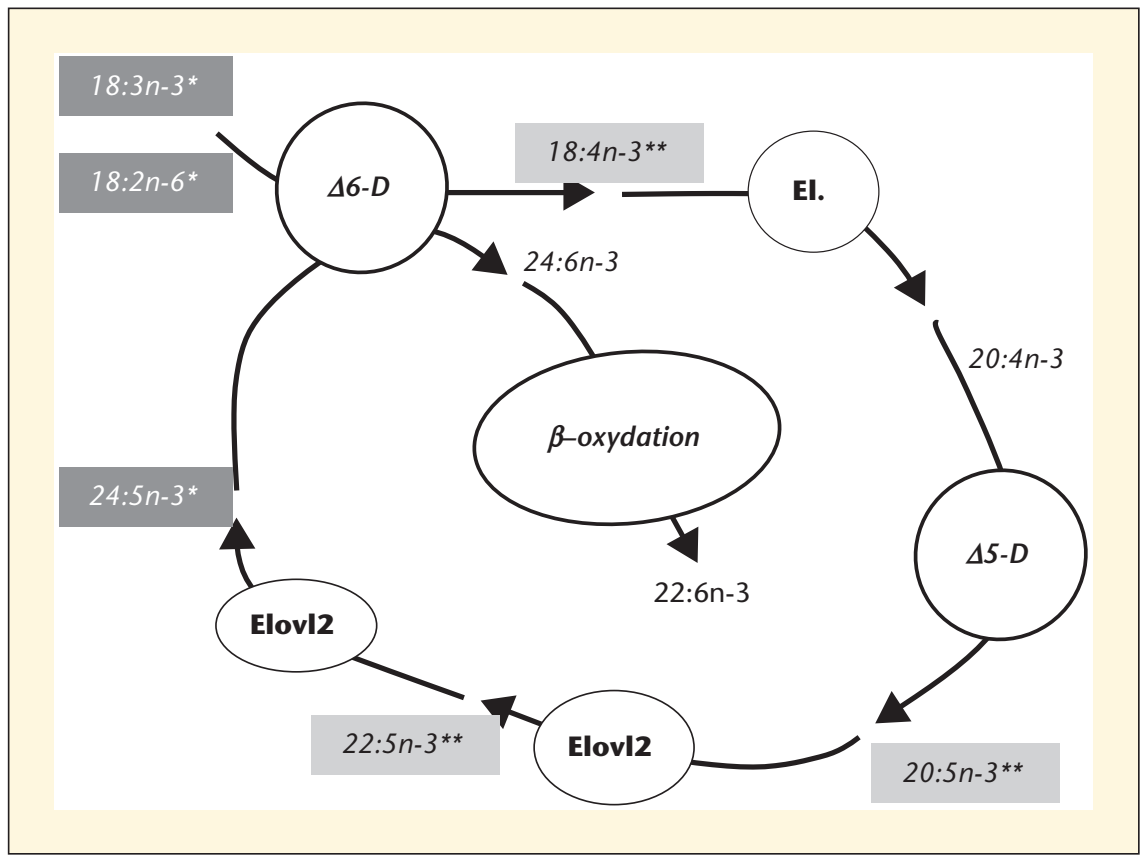

Figure 3. Voie de bioconversion de l'acide $\alpha$-linolénique (18:3n-3) en acide docosahexaénoïque (DHA, 22:6n-3). Étapes pour lesquelles une régulation de type inhibition compétitive a été démontrée (adapté de Gibson et al., 2011, et de Grégory et al., 2011). * Inhibition compétitive au niveau de la $\Delta 6$ désaturase; ** Inhibition compétitive au niveau de l'élongase Elovl2. El. : élongase ; D6-D : D6 désaturase; D5-D : D5 désaturase.

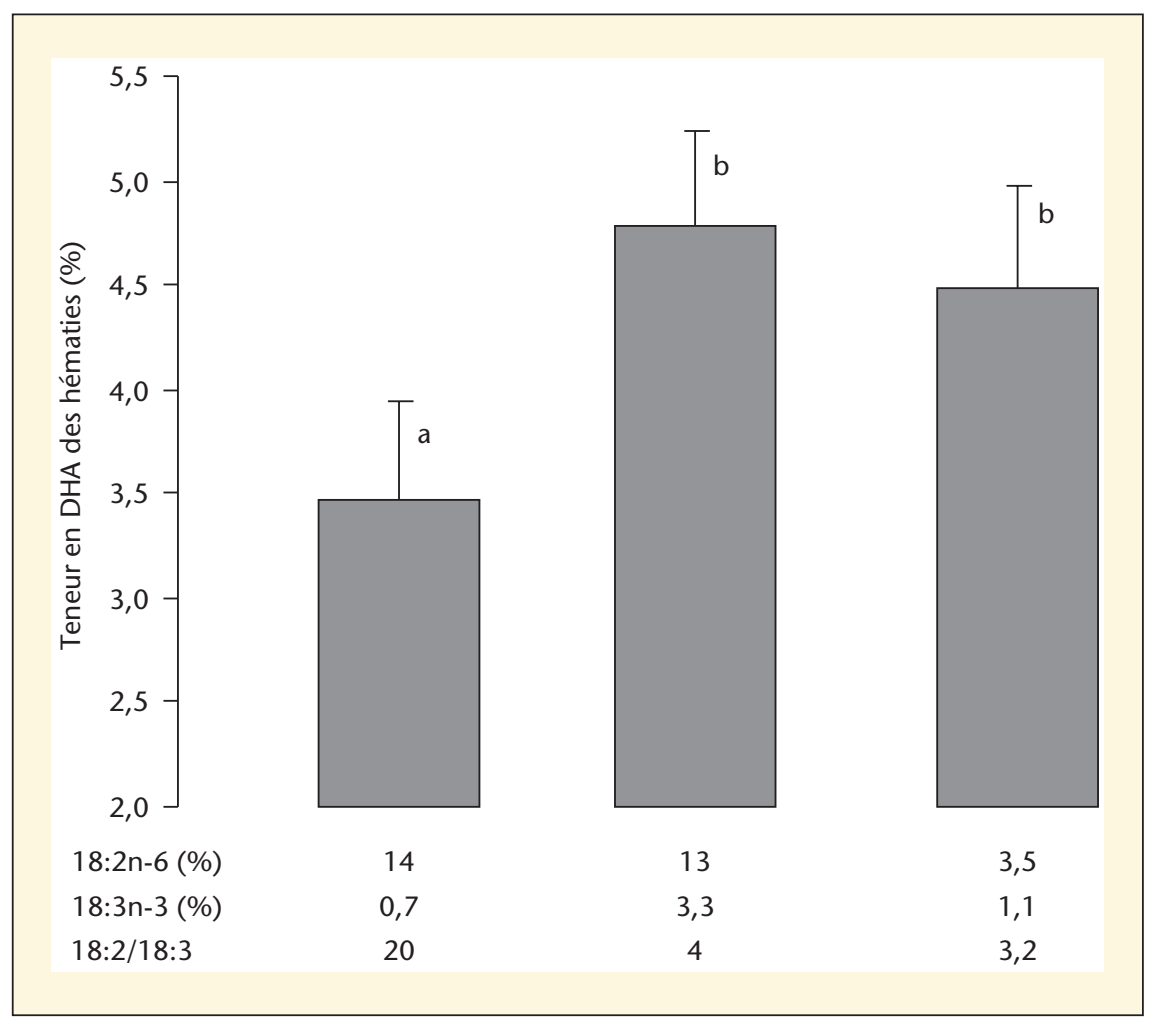

Figure 4. Impact des teneurs en acides linoléique (18:2n-6) et $\alpha$-linolénique (18:3n-3) (\% des acides gras totaux) de l'alimentation lactée sur la teneur en acide docosahexaénoïque (22:6n3) des hématies du nourrisson né à terme (Clark et al., 1992). ${ }^{a},{ }^{b}$ les groupes ne comportant pas de lettres communes sont significativement différents. 
sein ou recevant du DHA préformé (Gale et al., 2010). Cependant, ces différences n'apparaissent plus lorsque les facteurs confondants sont pris en compte dans I'analyse statistique (QI maternel, niveau d'éducation, classe sociale).

\section{Nutrition maternelle - Équilibre n-6/n-3 et obésité de I'enfant}

Des données épidémiologiques américaines suggèrent que l'augmentation de l'index d'adiposité du jeune enfant constatée au cours de ces 40 dernières années pourrait être liée à des variations de la qualité des acides gras alimentaires ingérés. L'installation progressive d'un déséquilibre alimentaire en AGPI au cours des dernières décennies aux USA, en faveur des AGPI n-6 et au détriment des AGPI n-3, pourrait expliquer cette tendance (Ailhaud et Guesnet, 2004 ; Ailhaud et al., 2006). En effet, dans ce pays, on dispose d'un nombre important de données de consommation qui ont permis de constater depuis les années 50 un accroissement progressif de la quantité d'acide linoléique consommé, passant de moins de $4 \%$ des calories totales ingérées à plus de $8 \%$ à partir des années 90. Cette évolution se reflète dans celle de la composition du lait maternel qui est un marqueur biologique fiable du niveau de consommation des populations (Guesnet et al., 2009). La teneur en acide linoléique s'est également accrue au cours de cette même période, avoisinant les 6-7 \% des acides gras totaux en 1950 pour atteindre une valeur maximale de 15-16\% dans les années $80-90$, soit près de 2 fois l'apport recommandé pour le nouveauné (Ailhaud et al., 2006). Pendant la même période, la teneur en acide $\alpha$ linolénique est restée stable, se traduisant par l'accroissement progressif du rapport $18: 2 n-6 / 18: 3 n-3$, lequel peut aujourd'hui atteindre des valeurs supérieures à 20 alors que le niveau recommandé est de 5 à 15 (ANC acides gras, 2011). Ces modifications proviennent de changements profonds de consommation alimentaire, impliquant les matières grasses visibles et cachées (matières grasses animales dont la composition a évolué). L'apport en acide arachidonique et en AGPI n-3 à longue chaîne (notamment le DHA) est également un facteur déterminant dans ce déséquilibre, aboutissant à une augmentation du rapport acide arachidonique/DHA dans les lipides circulants (Ailhaud et al., 2008). L'installation progressive de ce déséquilibre semble être une tendance générale dans les pays industrialisés car elle se manifeste également en Angleterre au niveau du lait maternel (Ailhaud et al., 2006). Dans $\mathrm{d}^{\prime}$ autres pays industrialisés pour lesquels nous ne disposons que de peu de données longitudinales (Canada, Australie et France par exemple), on retrouve également une augmentation de la teneur en acide linoléique mais qui semble moins marquée en raison de l'absence de données expérimentale avant les années 70 (figure 5). Dans les années 90 en France, le rapport 18:2n-6/ 18:3n-3 pouvait être supérieur à 20 dans près d'un quart de la population étudiée et s'accompagnait d'une teneur basse en DHA (Guesnet et al., 1995).

Or, des études in vitro et in vivo conduites notamment par l'équipe de G. Ailhaud ont démontré que les AGPI n-6, à travers son principal représentant à longue chaîne l'acide arachidonique, sont de puissants activateurs de la différenciation et du développement du tissu adipeux au cours de la période de grossesse et d'allaitement, contrairement aux AGPI n-3 qui inhiberaient ce développement en s'opposant aux effets des $n-6$. Les travaux réalisés sur des préadipocytes murins ont montré que l'acide arachidonique joue un rôle d'hormone adipogénique en stimulant la formation d'adipocytes à partir de cellules précurseurs via son dérivé oxygéné la prostacycline 12 (Massiera et al., 2003). Les travaux conduits chez la souris ont mis en évidence que la consommation pendant plusieurs générations d'un régime hyperlipidique riche en acide linoléique et pauvre en acide $\alpha$-linolénique (15\% $\mathrm{d}^{\prime}$ huile de maïs) provoque, par rapport à un régime témoin, une augmentation de 40 à $50 \%$ de la masse adipeuse après le sevrage, qui se maintient ensuite à l'âge adulte (Massiera et al., 2003). Cette obésité est de nature transgénérationnelle car elle perdure pendant au moins 4 générations, et est associée à des troubles métaboliques comme l'insulino-résistance et l'activation de gènes pro-inflammatoires impliqués dans l'obésité (Massiéra et al., 2010). L'augmentation de la masse adipeuse est complètement abolie chez la souris transgénique dont le gène codant pour le récepteur à la prostacycline est invalidé, ou si l'alimentation apporte de l'acide $\alpha$ linolénique en quantité équilibrée (Massiera et al., 2003). Elle est liée à une augmentation du rapport 20:4n-6/AGPI n3 à longue chaîne dans le lait maternel et plusieurs tissus, et est également retrouvée sur d'autres modèles animaux recevant pendant la période périnatale des apports déséquilibrés en AGPI (Pouteau et al., 2010).

Le faible nombre d'études prospectives d'observation sur cohortes soulignent également un lien possible entre la consommation d'AGPI n-6 et le développement de l'obésité chez l'enfant.

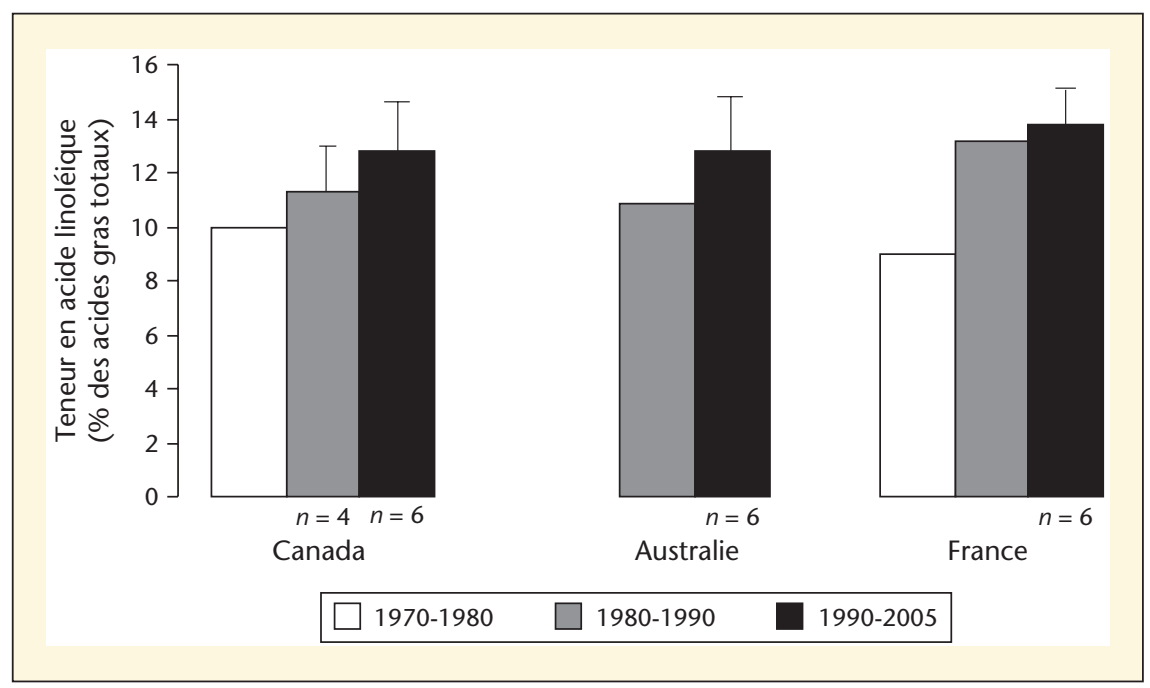

Figure 5. Évolution de la concentration de l'acide linoléique dans le lait maternel humain au cours des 4 dernières décennies au Canada, en Australie et en France (adapté d'Ailhaud et al., 2006). $\mathrm{n}=$ nombre d'etudes publiées. 
Dans l'étude Projet Viva conduite aux États-Unis sur 1250 couples mère-enfant suivis entre le dernier trimestre de grossesse et l'âge de 3 ans, une corrélation négative a été mise en évidence entre le statut en AGPI n-3 à longue chaîne à la $29^{\mathrm{e}}$ semaine post-conception et au cordon (niveau de consommation et/ou concentration circulante) avec l'épaisseurs des plis cutanés à l'âge de 3 ans (Donahue et al., 2011). Cette corrélation est également significative avec les rapports $n-6 / n-3$ et acide arachidonique/EPA+DHA. Une seconde étude prospective vient d'être publiée, confirmant la relation entre les AGPI n-6 et obésité (Moon et al., 2013). Cette étude anglaise (The Southampton Women's Survey) a été réalisée avec 300 couples mère-enfant, en mesurant le lien qui pouvait exister entre la concentration plasmatique maternelle en AGPI en fin de grossesse ( $34^{\mathrm{e}}$ semaine post-conception) et la composition corporelle de l'enfant à l'âge de 4 à 6 ans. Les données montrent clairement que la teneur plasmatique en AGPI n-6 est associée positivement au niveau d'adiposité de l'enfant à l'âge de 4 et 6 ans. L'association concerne à la fois I'acide linoléique et l'acide arachidonique et est toujours significative après ajustement aux facteurs de confusion (durée de I'allaitement maternel, tabac, BMI des mères, âge des mères à la naissance, niveau de consommation en énergie en lipides et en sucres, etc.), mais elle ne concerne pas les AGPI n-3. Cette étude suggère donc qu'une réduction des apports maternels en AGPI n-6 serait une approche nutritionnelle plus efficace pour réduire le risque d'obésité chez l'enfant que l'augmentation des apports en AGPI n-3. Cependant, une dernière étude menée chez des femmes allaitantes indique que la teneur en DHA du lait maternel est négativement associée avec I'adiposité de l'enfant entre les âges de 2 et 7 ans (Pedersen et al., 2012).

Les quelques études d'intervention nutritionnelle publiées à ce jour fournissent des résultats peu concluants et ont exclusivement porté sur les effets d'une supplémentation du régime maternel (fin de grossesse - période d'allaitement) avec des doses d'AGPI n-3 à longue chaîne $s^{\prime}$ échelonnant entre 0,2 et 1,2 g/jour (Muhlhausler et al., 2013). Les réserves que I'on peut émettre sur ces études portent notamment sur le niveau de base moyen de consommation en AGPI qui semble adéquat (et donc non déséquilibré) dans la popula- tion supplémentée (Hauner et al., 2012). Rappelons que la situation de déséquilibre en AGPI typique des années 1990-2000 semble s'être significativement améliorée au cours de ces 10 dernières années. Elle résulte notamment de l'augmentation de l'offre proposée à la consommation d'acide $\alpha$ linolénique par les industriels résultant de l'évolution des connaissances et des recommandations nutritionnelles. Une étude réalisée sur le lait maternel illustre bien cette évolution en France (BouéVaysse et al., 2009). Les données recueillies dégagent une tendance marquée à la baisse de la teneur en acide linoléique (- $20 \%$ ) et surtout une augmentation significative (+ $30 \%$ ) de celle de l'acide $\alpha$-linolénique (figure 6 ). Ces variations opposées contribuent ainsi à limiter le déséquilibre entre les AGPI n-6 et n-3. La consommation plus importante $d$ 'huiles riches en acide $\alpha$ linolénique sous la forme visible (huile de colza par exemple) et invisible (produits élaborés) a vraisemblablement contribué à améliorer de façon significative le statut en AGPI de la population française. Cependant le niveau actuel de consommation quotidienne en cet AGPI n-3 par la femme allaitante reste encore à améliorer en regard de ses besoins physiologiques, ainsi que des besoins plus spécifiques de l'enfant allaité au sein en regard de son développement et de la protection contre certaines maladies métaboliques de l'âge adulte.

\section{Conclusion}

Il apparaît maintenant important de développer des recherches visant à confirmer le rôle exercé par les caractéristiques structurales uniques du lait maternel sur le développement du syndrome métabolique et de l'obésité (taille, membrane phospholipidique). La position préférentielle du 16:0 en position interne, et par voie de conséquence la localisation des autres acides gras majeurs en position externe (acide oléique et AGPI essentiels), apparaît un paramètre important contrôlant les processus d'absorption intestinal des acides gras et de leur devenir au niveau tissulaire qu'il conviendrait aussi d'explorer (Innis, 2011).

On sait que l'apport alimentaire en acides linoléique et $\alpha$-linolénique et le rapport linoléique/ $\alpha$-linolénique sont des paramètres déterminants du métabolisme et du niveau d'incorporation tissulaire du DHA. Pour limiter les phénomènes $d$ 'inhibition compétitive au niveau de la voie de bioconversion et donc maintenir un équilibre $n-6 / n-3$ convenable, il semble plus judicieux de limiter les teneurs en ces AGPI essentiels dans les laits infantiles plutôt que $d^{\prime}$ augmenter, en raison de teneurs élevées d'acide linoléique, celle de l'acide $\alpha$-linoléique (Gibson et al., 2011). Par ailleurs des études complémentaires seront nécessaires pour fournir des informations précises sur les conséquences neurofonctionnelles à

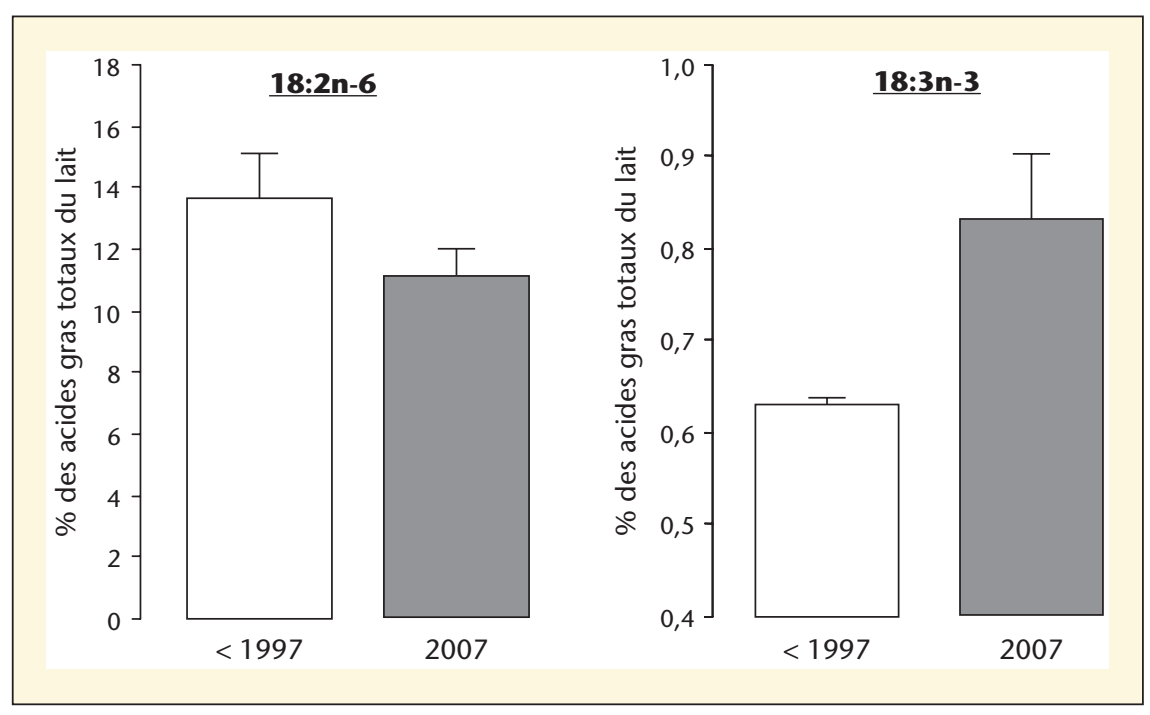

Figure 6. Evolution des concentrations en AGPI précurseurs dans le lait maternel en France entre 1997 et 2007 (adapté de Boué-Vaysse et al., 2009). 
long terme d'une supplémentation en $\mathrm{DHA}$, mais également sur un besoin spécifique en DHA après les 6 premiers mois de la vie.

Concernant l'exposition pré- et postnatale en AGPI et le développement de l'obésité chez l'enfant, les données animales et d'observation démontrent qu'un statut materno-fœtal en n-6 élevé (ou déséquilibré dans le rapport n-6/ n-3) est associé avec une plus grande adiposité chez l'enfant. Des études cliniques d'intervention nutritionnelle visant, soit à supplémenter en AGPI n-3 à longue chaîne des populations fortes consommatrices d'AGPI n-6, soit à réduire par des conseils diététiques les apports en AGPI n-6, demandent à être réalisées pour confirmer les premières données expérimentales (Moon et al., 2013 ; Muhlhausler et al., 2013). Elles devront au préalable évaluer le statut circulant et le niveau de consommation en AGPI des populations étudiées.

Remerciements. Philippe Guesnet dédicace cet article au Pr R.G. Jensen qui a été à l'initiative des travaux conduits sur le lait maternel humain. II remercie également la société PG Consulting pour son soutien financier.

\section{Conflits d'intérêts : aucun}

\section{RÉFÉRENCES}

Ailhaud G, Guesnet P. Fatty acid composition of fats is an early determinant of childhood obesity : a short review and an opinion. Obes Rev 2004 ; 5 : 21-6.

Ailhaud G, Massiera F, Weill P, Legrand P, Alessandri JM, Guesnet $P$. Temporal changes in dietary fats : role of $n-6$ polyunsaturated fatty acids in excessive adipose tissue development and relationship to obesity. Prog Lipid Res 2006 ; 45 : 203-36.

Ailhaud G, Guesnet P, Cunnane SC. An emerging risk factor for obesity : does disequilibrium of polyunsaturated fatty acid metabolism contribute to excessive adipose tissue development? Br J Nutr 2008 ; 100 : 461-70.

ANC acides gras, 2011. Actualisation des apports nutritionnels conseillés pour les acides gras. Rapport d'expertise collective, Comité d'Experts Spécialisé Nutrition humaine, Groupe de travail ANC acides gras, http://pmb.santenpdc.org/opac_css/doc_num.php?explnum_id=11878.

Alessandri JM, Guesnet P, Vancassel S, et al. Polyunsaturated fatty acids in the central nervous system: evolution of concepts and nutritional implications throughout life. Reprod Nutr Dev 2004 ; 44 : 509-38.

Armand M, Hamosh M, Mehta NR, et al. Effect of human milk or formula on gastric function and fat digestion in the premature infant. Pediatr Res 1996 ; 40 : 429-37.

Armand $M$, Pasquier $B$, André $M$, et al. Digestion and absorption of 2 fat emulsions with different droplet sizes in the human digestive tract. Am / Clin Nutr $1999 ; 70$ : 1096-106.

Beyerlein A, von Kries R. Breastfeeding and body composition in children: will there ever be conclusive empirical evidence for a protective effect against overweight? Am J Clin Nutr 2011 ; 94 : 1772S-5.

Borel P, Armand $\mathrm{M}$, Pasquier B, et al. Digestion and absorption of tube-feeding emulsions with different droplet sizes and compositions in the rat. J Parenter Enteral Nutr $1994 ; 18: 534-43$.

Boué-Vaysse C, Billeaud C, Guesnet Ph, Couëdelo L, Alessandri JM, Putet G, Combe $N$. Teneurs en acides gras polyinsaturés essentiels du lait maternel en France : évolution du contenu en acides linoléique et alpha-linolénique au cours des 10 dernières années. OCL 2009 ; 16 : 4-7.

Bourre JM, Francois M, Youyou A, Dumont O, Piciotti M, Pascal G, Durand G. The effects of dietary alpha-linolenic acid on the composition of nerve membranes, enzymatic activity, amplitude of electrophysiological parameters, resistance to poisons and performance of learning tasks in rats. J Nutr $1989 ; 119$ : 1880-92.

Bourre JM, Piciotti M, Dumont O, Pascal G, Durand G. Dietary linoleic acid and polyunsaturated fatty acids in rat brain and other organs. Minimal requirements of linoleic acid. Lipids $1990 ; 25: 465-72$.

Carnielli VP, Luijendijk IH, van Beek RH, Boerma G], Degenhart HJ, Sauer PJ. Effect of dietary triacylglycerol fatty acid positional distribution on plasma lipid classes and their fatty acid composition in preterm infants. Am J Clin Nutr $1995 ; 62$ : 776-81.

Clark KJ, Makrides M, Neumann MA, Gibson RA. Determination of the optimal ratio of linoleic acid to $\alpha$-linolenic acid in infant formulas. J Pediatr $1992 ; 120$ : S151-8.

Cunnane SC. Problems with essential fatty acids : time for a new paradigm? Prog Lipid Res 2003 ; 42 : 544-68.

Cunnane SC, Guesnet P. Linoleic acid recommendations - A house of cards. Prostaglandins Leukot Essent Fatty Acids 2011 ; 85 : 399-402.

Delplanque B, Du Q, Agnani G, Le Ruyet P, Martin JC. A dairy fat matrix providing alphalinolenic acid (ALA) is better than a vegetable fat mixture to increase brain DHA accretion in young rats. Prostaglandins Leukot Essent Fatty Acids 2013 ; 88 : 115-20.

Donahue SM, Rifas-Shiman SL, Gold DR, Jouni ZE, Gillman MW, Oken E. Prenatal fatty acid status and child adiposity at age $3 \mathrm{y}$ : results from a US pregnancy cohort. Am J Clin Nutr $2011 ; 93$ : 780-8.

Du Q, Martin JC, Agnani G, Pages N, Leruyet P, Carayon P, Delplanque B. Dairy fat blends high in $\alpha$-linolenic acid are superior to $n-3$ fatty-acid-enriched palm oil blends for increasing DHA levels in the brains of young rats. J Nutr Biochem 2012 ; 23 : 1573-82.

Gale CR, Marriott LD, Martyn CN, Group for Southampton Women's Survey Study et al. Breastfeeding, the use of docosahexaenoic acid-fortified formulas in infancy and neuropsychological function in childhood. Arch Dis Child $2010 ; 95$ : 174-9.

Gibson RA, Muhlhausler B, Makrides M. Conversion of linoleic acid and alpha-linolenic acid to long-chain polyunsaturated fatty acids (LCPUFAs), with a focus on pregnancy, lactation and the first 2 years of life. Matern Child Nutr $2011 ; 7$ : 17-26.

Gregory MK, Gibson RA, Cook-Johnson RJ, Cleland LG, James MJ. Elongase reactions as control points in long-chain polyunsaturated fatty acid synthesis. PLoS One 2011 ; 6 : e29662.

Guesnet P, Antoine JM, Rochette de Lempdes JB, Galent A, Durand G. Polyunsaturated fatty acid composition of human milk in France: changes during the course of lactation and regional differences. Eur / Clin Nutr 1993 ; 47 : 700-10.

Guesnet P, Couet C, Alessandri JM, Antoine JM, Durand G. Variabilité de la teneur en acide linoléique $(18: 2 n-6)$ et du rapport 18 : 2n6/18 : 3n-3 des lipides dans le lait de femme en France. Ann Pédiatrie (Paris) 1995 ; 42 : 282-8.

Guesnet P, Combe N, Ailhaud G, Alessandri JM. La teneur en acides gras polyinsaturés du lait maternel : un marqueur biologique fiable du niveau de consommation des populations. OCL $2009 ; 16: 1-3$.

Guesnet P, Lallemand SM, Alessandri JM, Jouin $\mathrm{M}$, Cunnane SC. $\alpha$-Linolenate reduces the dietary requirement for linoleate in the growing rat. Prostaglandins Leukot Essent Fatty Acids $2011 ; 85$ : 353-60.

Guesnet P, Alessandri JM. Docosahexaenoic acid (DHA) and the developing central nervous system (CNS) - Implications for dietary recommendations. Biochimie 2011 ; $93: 7-12$.

Hauner $\mathrm{H}$, Much D, Vollhardt C, et al. Effect of reducing the $n-6: n-3$ long-chain PUFA ratio 
during pregnancy and lactation on infant adipose tissue growth within the first year of life : an open-label randomized controlled trial. Am J Clin Nutr 2012 ; 95 : 383-94.

Holman RT. Biological activities of polyunsaturated fatty acids. Prog Chem fats lipids 1970 ; IX : 607-82.

Jensen RG. The lipids in human milk. Prog Lipid Res 1996 ; 35 : 53-92.

Innis SM, Dyer R, Quinlan P, Diersen-Schade D. Palmitic acid is absorbed as sn-2 monopalmitin from milk and formula with rearranged triacylglycerols and results in increased plasma triglyceride sn- 2 and cholesteryl ester palmitate in piglets. I Nutr $1995 ; 125: 73-81$.

Innis SM. Dietary triacylglycerol structure and its role in infant nutrition. Adv Nutr $2011 ; 2$ : 275-83.

Kennedy K, Fewtrell MS, Morley R, et al. Double-blind, randomized trial of a synthetic triacylglycerol in formula-fed term infants: effects on stool biochemistry, stool characteristics, and bone mineralization. Am / Clin Nutr $1999 ; 70$ : 920-7.

Kramer MS. Breastfeeding and allergy: the evidence. Ann Nutr Metab 2011 ; 59 : 20-6.

Massiera F, Saint-Marc P, Seydoux J, et al. Arachidonic acid and prostacyclin signaling promote adipose tissue development: a human health concern? J Lipid Res 2003 ; 44 : 271-9.
Massiera F, Barbry P, Guesnet P, et al. A Western-like fat diet is sufficient to induce a gradual enhancement in fat mass over generations. J Lipid Res 2010 ; 51 : 2352-61.

Michalski MC, Briard V, Michel F, Tasson F, Poulain P. Size distribution of fat globules in human colostrum, breast milk, and infant formula. J Dairy Sci 2005 ; 88 : 1927-40.

Michalski MC, Briard V, Desage M, Geloen A. The dispersion state of milk fat influences triglyceride metabolism in the rat - $\mathrm{A}{ }^{13} \mathrm{CO} 2$ breath test study. Eur J Nutr 2005 ; 44 : 43644.

Michalski MC, Soares AF, Lopez C, Leconte N, Briard V, Geloen A. The supramolecular structure of milk fat influences plasma triacylglycerols and fatty acid profile in the rat. Eur J Nutr 2006 ; 45 : 215-24.

Moon RJ, Harvey NC, Robinson SM, et al. Maternal plasma polyunsaturated fatty acid status in late pregnancy is associated with offspring body composition in childhood. I Clin Endocrinol Metab 2013 ; 98 : 299-307.

Muhlhausler BS, Ailhaud GP. Omega-6 polyunsaturated fatty acids and the early origins of obesity. Curr Opin Endocrinol Diabetes Obes $2013 ; 20$ : 56-61.

Oosting A, Kegler D, Wopereis HJ, et al. Size and phospholipid coating of lipid droplets in the diet of young mice modify body fat accumulation in adulthood. Pediatr Res $2012 ; 72$ : 362-9.
Owen CG, Whincup PH, Cook DG. Breastfeeding and cardiovascular risk factors and outcomes in later life: evidence from epidemiological studies. Proc Nutr Soc $2011 ; 70$ : 478-84.

Pedersen L, Lauritzen L, Brasholt M, Buhl T, Bisgaard $H$. Polyunsaturated fatty acid content of mother's milk is associated with childhood body composition. Pediatr Res 2012 ; 72 : 631-6.

Pouteau E, Aprikian O, Grenot C, et al. A low alpha-linolenic intake during early life increases adiposity in the adult guinea pig. Nutr Metab (Lond) 2010 ; doi : 10.1186/17437075r-r7-8.

Salle B. L'alimentation du nouveau né et du nourrisson. Bull Acad Natle Méd 2009 ; 193 :

Uauy RD, Birch DG, Birch EE, Tyson JE, Hoffman DR. Effect of dietary omega-3 fatty acids on retinal function of very-low-birthweight neonates. Pediatr Res $1990 ; 28$ : 48592.

van Greevenbroek MM, Voorhout WF, Erkelens DW, van Meer G, de Bruin TW. Palmitic acid and linoleic acid metabolism in Caco-2 cells different triglyceride synthesis and lipoprotein secretion. J Lipid Res 1995 ; 36 : 13-24.

Yao Y, Eshun JK, Lu S, Berschneider HM, Black DD. Regulation of triacylglycerol and phospholipid trafficking by fatty acids in newborn swine enterocytes. Am J Physiol Gastrointest Liver Physiol 2002 ; 282 : G817-24. 